\title{
USO DE Bacillus thuringiensis (H-14) COMO AGENTE DE CONTROLE DE MOSQUITOS EM UM CEMITÉRIO
}

\author{
Luciana Urbano dos Santos.., Álvaro Braga de Souza", Carlos Fernando \\ Salgueirosa de Andrade "* \& Carlos Eduardo Pilleggi de Souza
}

\section{RESUMO}

Cemitérios são pontos críticos quanto ao controle de vetores da Dengue devido à utilização de vasos e floreiras em túmulos, que possibilitam o acúmulo de água formando habitat propício para os mosquitos. O presente trabalho avaliou a eficiência do emprego de Bacillus thuringiensis var. israelensis (H-14) no controle de culicídeos nesses criadouros, associado ao desempenho de uma equipe municipal de Saúde Pública. Com relação aos métodos convencionais (químico e mecânico) os resultados obtidos indicam vantagens na utilização do método biológico executado pelas equipes de Saúde Pública no manejo desses culicídeos. O emprego do Bacillus thuringiensis (H-14) mostrou-se seguro e econômico Apenas com um treinamento rápido e pouca mudança na rotina das atividades da equipe municipal considerada, foi obtido mais de $99 \%$ de eficiência após uma série de três aplicaçø̃es.

UNITERMOS: Controle de mosquito, Cemitério, Bacillus thuringiensis (H-14).

\section{INTRODUÇÃO}

A Prefeitura Municipal de Campinas (PMC) e a Superintendência de Controle de Endemias (SUCEN) têm realizado, nos últimos anos, esforços no sentido de

\footnotetext{
- Pós-Graduação Depto. de Parasitologia - Inst.de Biologia da Universidade Estadual de Campinas, Campinas,São Paulo,Brasil

.. Prefeitura Municipal de Campinas, Campinas, São Paulo, Brasil

-.. Depto. de Zoologia - Inst. de Zoologia da Universidade Estadual de Campinas, São Paulo, Brasil

Endereço para Correspondência: Depto. Zoologia/IB/UNICAMP.Caixa Postal 6109. CEP: 13081 970. Campinas, São Paulo, Brasil

Recebido para publicação $\mathrm{em} \mathrm{30/09/94}$
} 
combater os pernilongos incriminados como vetores da dengue e febre amarela urbana, Aedes aegypti (Linn.) e Ae.albopictus (Skuse), para reduzir o risco de epidemias na região. A infestação por esses culicídios em áreas urbanas dos municípios do Estado de São Paulo tem sido crescente, e casos de dengue têm sido registrados pela imprensa em municípios relativamente próximos como Marilia, Barra Bonita, Bauru ${ }^{(4)}$

Cemitérios são reconhecidamente pontos críticos nos programas de controle dos vetores da dengue em vários países ${ }^{(11,12,14)} \mathrm{A}$ água de chuva acumulada em vasos $\mathrm{e}$ floreiras normalmente se constitui em criadouro propício para os mosquitos vetores.

Muitas vezes ainda, e devido à decomposição de folhas caídas das árvores ou das flores que não são recolhidas após murcharem, a água desses recipientes acumulam elevado teor de matéria orgânica, constituindo-se por outro lado em adequado criadouro de espécies de Culex, principalmente a espécie urbana Culex quinquefasciatus.

Em Campinas, a lei municipal No.6756/91 obriga os proprietários de túmulo a colocarem areia nos vasos e floreiras. Apesar da própria PMC iniciar esse serviço em 1991, a grande maioria dos proprietários removeram a areia e novamente colocaram água nos vasos: insensíveis ou desinformados quanto ao problema que isso pode causar. Esse método porém mostrou-se eficiente em Porto Rico onde 26 cemitérios tiveram larvas de Ae.aegypti controladas por quatro anos, com a utilização, associada a mensagens de educação para a população ${ }^{(10)}$.

Como a fiscalização permanente em cemitérios, de uma forma geral, é difícil, métodos alternativos de controle vêm sendo avaliados no município de Campinas.

A possibilidade de se conseguir o controle de espécies de Aedes com o uso de agentes biológicos tem sido indicada em diversos trabalhos. Entre esses agentes estão predadores como copépodos ciclopóideos ${ }^{(7,13)}$; planárias ${ }^{(3,9)}$ e mosquitos Toxorhynchitinae ${ }^{(2,5)}$, e ainda patógenos como fungos ${ }^{(8)} \mathrm{e}$ bactérias como Bacillus sphaericus e B.thuringiensis var.israelensis $(\mathrm{BTI})^{(1,6)}$.

No Brasil, apenas essa última bactéria encontra-se no mercado na forma de produto comercial.

O presente trabalho trata de avaliar a eficiência da equipe municipal da PMC no controle de Aedes, $\boldsymbol{e}$ de uma série de 3 aplicações do produto Teknar, à base de BTI no combate aos culicídeos do Cemitério da Saudade, Campinas-SP.

\section{MATERIAIS E MÉTODOS}

A aplicação do Bacillus thuringiensis (H-14) - O Cemitério da Saudade Localiza-se em zona urbana de Campinas-SP (Bairro Swift). Sua área é de $180.000 \mathrm{~m} 2$ dividida em 78 quadras e abrigando aproximadamente 28.000 túmulos.
SANTOS,LU.SOUZA,A B.ANDRADE,CF S. \& SOUZA,CEP. Uso de Bacillus thuringiensis como Agente de controle de Mosquitos em um Cemitério.Rev.Pat.Trop.23 ( 2 ):151-158 jul./dez.1994

Para efeito de divisão das tarefas de aplicação e avaliação, a área total do cemitério foi subdividida em 05 setores utilizando-se como critério um número aproximadamente igual de quadras por setor. (FIGURA.1).

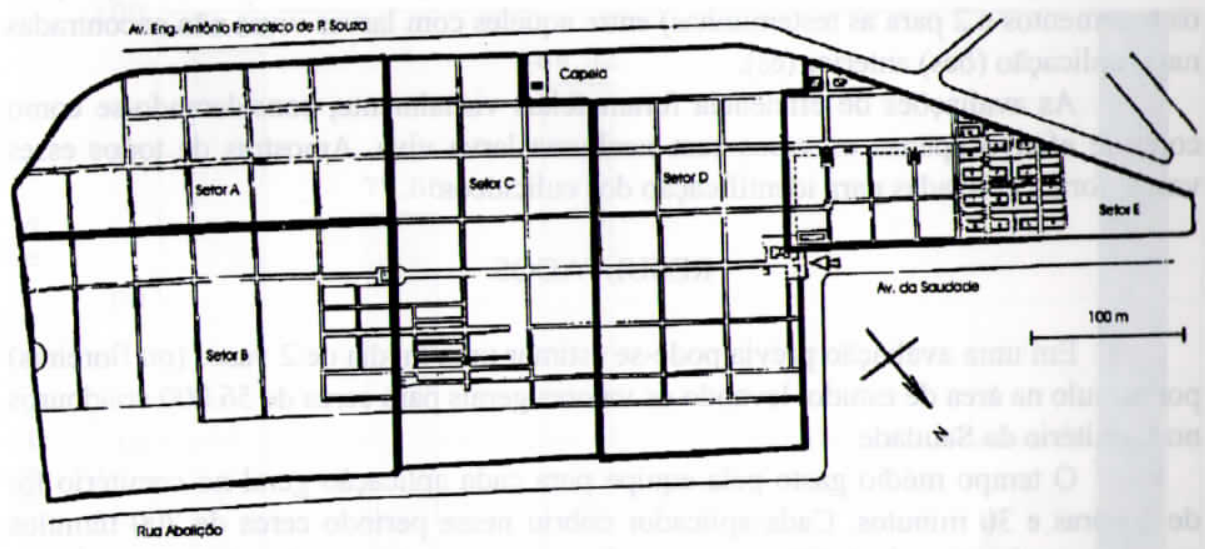

Fig.1 - Aspecto Geral do Cemitério da Saudade (Campinas/SP) com as divisð̃es dos setores usados para a avaliação

Para o tratamento dos criadouros utilizou-se o larvicida comercial Teknar 3,000 AAU, (com 1200 ITU/mg de potência). A concentração operacional foi de 5.000 ITU/ml de água, calculada para uma estimativa de dois litros de água em média contida nos vasos. Foram usados aplicadores manuais com capacidade para $500 \mathrm{ml}$, com jato grosso, calibrados previamente de forma que um único espirro (cerca de $1 \mathrm{ml}$ ) contivesse a concentração operacional para 2 litros de água do criadouro.

Foram realizados três tratamentos gerais com um intervalo de 7 dias entre eles, procurando-se cobrir todos os criadouros do cemitério. Os funcionários da equipe municipal de controle de Aedes, receberam apenas um treinamento prévio para esse trabalho. As equipes foram compostas de : 2 Coordenadores Gerais, 5 Supervisores ( 1 para cada setor), 40 aplicadores ( 8 para cada setor) e 1 ajudante geral para fazer recarregamento dos pulverizadores manuais e preparo da concentração estoque (larvicida + água). Os grupos de aplicadores trataram seus setores caminhando no mesmo sentido e direção. Cada aplicador tratava os criadouros das quadras na sua área andando em zig-zag e aplicando diretamente o produto na água contida nos vasos ou floreiras. Em casos especiais onde o volume líquido no criadouro era visivelmente 
SANTOS,L.U.;SOUZA,A.B.;ANDRADE,C.F.S. \& SOUZA,C.E.P. Uso de Bacillus thuringiensis como Agente de controle de Mosquitos em um Cemitério.Rev.Pat.Trop.23 ( 2):151-158,jul./dez.1994

maior que dois litros, ou continha muita matéria orgânica, os aplicadores foram instruídos para aplicar dois espirros ( cerca de $2 \mathrm{ml}$ ).

Avaliação - Vinte e quatro horas antes da primeira aplicação foram tomados aleatoriamente 14 vasos que contivessem larvas, em cada setor, sendo que 2 deles foram cobertos com filó e não receberam os tratamentos, servindo como testemunhas da $m$ - talidade natural. Os 12 vasos restantes foram discretamente numerados e mapeados para avaliação dos tratamentos, 24 horas após essa primeira aplicação. Para avaliar a segunda e terceira aplicações, foi tomado um igual número de vasos (12 para os tratamentos e 2 para as testemunhas) entre aqueles com larvas vivas não encontradas na(s) aplicação (ões) anterior (es).

As avaliações de eficiência foram feitas visualmente, considerando-se como controle efetivo apenas os vasos sem nenhuma larva viva. Amostras de todos esses vasos, foram coletadas para identificação dos culicídeos.

\section{RESULTADOS}

Em uma avaliação prévia pode-se estimar uma média de 2 vasos (ou floreiras) por túmulo na área de estudo, levando os valores gerais para cerca de 56.000 criadouros no Cemitério da Saudade.

O tempo médio gasto pela equipe para cada aplicação geral no cemitério foi de 2 horas e 30 minutos. Cada aplicador cobriu nesse período cerca de 700 túmulos com uma estimativa de 1.400 vasos tratados, recarregando suas bombas manuais em geral três vezes. O volume total de produto comercial gasto foi de aproximadamente $900 \mathrm{ml}$

A análise das larvas coletadas nos vasos usados para as três avaliações indicou uma predominância média de Culex quinquefasciatus ocorrendo em $72,8 \%$ do recipientes. Em 3,9\% dos vasos ocorreram larvas de Aedes albopictus e em 8,7\% ocorreram larvas de outros culicídeos. Uma média ainda de $14,5 \%$ dos vasos estavam secos, alguns caídos, ou encontravam-se alterados não permitindo a coleta de larvas. (TABELA 1).

TABELA 1. Porcentagem relativa nédia de ocorrência de pernilongos, nos cinco setores do Cemitério da Saudade, para três avaliaçð̃es.

\begin{tabular}{ccccc}
\hline Avaliações & $\begin{array}{c}\text { Culex } \\
\text { quinquesfaciatus }\end{array}$ & $\begin{array}{c}\text { Aedes } \\
\text { albopictus }\end{array}$ & $\begin{array}{c}\text { Outros } \\
\text { Culicídeos }\end{array}$ & $\begin{array}{c}\text { Vasos } \\
\text { alterados }\end{array}$ \\
\hline $1^{\mathrm{a}}$ & $72,8 \%$ & $4,3 \%$ & $11,4 \%$ & $11,4 \%$ \\
$2^{\mathrm{a}}$ & $74,2 \%$ & $6,1 \%$ & $6,0 \%$ & $13,6 \%$ \\
$3^{\mathrm{a}}$ & $71,4 \%$ & $1,5 \%$ & $8,5 \%$ & $18,5 \%$ \\
\hline
\end{tabular}

SANTOS,L.U.;SOUZA,A.B.;ANDRADE,C.F.S. \& SOUZA,C.E.P. Uso de Bacillus thuringiensis como Agente de controle de Mosquitos em um Cemitério.Rev.Pat.Trop.23 (2):151-158.jul./dez.1994

Após a primeira aplicação, a eficiência de controle nos cinco setores variou entre $81,8 \%$ a $100 \%$, com uma média de $94,5 \%$. A segunda aplicação, uma semana após, permitiu por si só eficiências entre $66,6 \%$ e $81,8 \%$, elevando a eficiência média de controle acumulada para $98,47 \%$. A última aplicação permitiu eficiências variando entre $81,8 \%$ a $100 \%$ elevando o acumulado final médio para $99,8 \%$ de controle. (FIGURA 2).

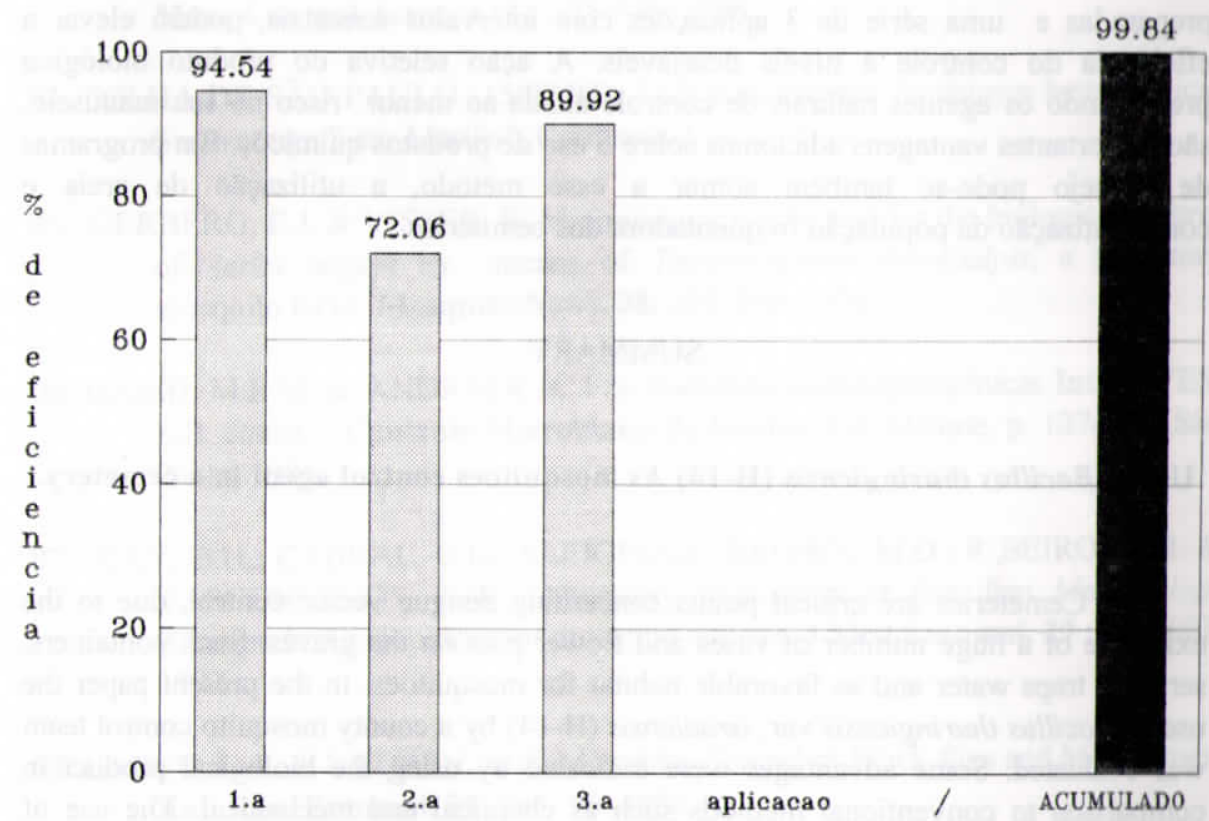

Fig.2 - Eficiência média de controle após cada aplicac̃o e acumulado final para uso de BTI no Cemiterio du Saudade.

\section{DISCUSSÃO}

A proporção relativa de criadouros contendo água com alto teor de matéria orgânica e larvas de Cx. quinquefasciatus foi bastante elevada. Se por um lado isso reduz os recursos para a criação dos vetores da dengue, reflete também um descuido quase geral por parte dos responsáveis pelos jazigos. Tal espécie, devido à sua pronunciada antropofilia contitui-se em grave incômodo à população dos bairros vizinhos ao cemitério. Com maior gravidade, uma média de $3,9 \%$ dos vasos ou floreiras contendo larvas de Ae. albocipctus poderiam representar para o cemitério 
cerca de 2.200 criadouros para os vetores da dengue; fato alarmante em termos epidemiológicos. Entre os outros culicídeos registrados, ocorreram espécies com pouca antropofilia como Ae. fluviatilis.

Devido ao baixo efeito residual dos formulados em suspensão aquosa de BTI, como é o caso de TEKNAR, e ainda às dificuldades de operacionalização dos tratam-..tos em ambientes como cemitérios, a eficiência de apenas uma aplicação pode ser baixa, da ordem de $65 \%$ como ocorreu um um setor. O trabalho de equipes melhor preparadas e uma série de 3 aplicaçø̃es com intervalos semanais, podem elevar a eficiência do controle a níveis desejáveis. A ação seletiva do produto biológico preservando os agentes naturais de controle aliada ao menor risco no seu manuseio, são importantes vantagens adicionais sobre o uso de produtos químicos. Em programas de manejo pode-se também somar a esse método, a utilização de areia e conscientização da população freqüentadora dos cemitérios.

\section{SUMMARY}

\section{Use of Bacillus thuringiensis (H-14) As mosquitoes control agent in a cemetery.}

Cemeteries are critical points concerning dengue vector control, due to the existence of a huge number of vases and flower pots on the graves. Such containers, serve as traps water and as favorable habitat for mosquitoes. In the present paper the use of Bacillus thuringiensis var. israelensis $(\mathrm{H}-14)$ by a county mosquito control team was evaluated. Some advantages were indicated by using the biological product in comparison to conventional methods such as chemical and mechanical. The use of Bacillus thuringiensis $(\mathrm{H}-14)$ showed to be of safe and economic. Whith just a quickly training and few changes in the work routine of the county team it was obtained more than of $99 \%$ efficiency after a series os 3 applications of BTI.

KEYWORDS: Mosquito control, Cemetery, Bacillus thuringiensis (H-14)

\section{REFERÊNCIAS BIBLIOGRÁFICAS}

01. ANDRADE,C.F.S. \& MODOLO,M. Susceptibility of Aedes aegypti larvae to Temephos and Bacillus thuringiensis var. israelensis in integrated control. Rev.Saúde Pública., 25 (3): 184-187. São Paulo, 1991.
02. BAYLEI, D.L.; RUSSEL, J.G. \& SIMMONDS, P.R. Effects of indigenous Toxorhychites rutillus rutillus on Aedes aegypti breeding in tire dumps. Mosquito News, 43: 33-37. 1983.

03. DARBY, W.N.; BOOBAR, L.R. \& SARDELES, M.R. A method for disopersing planaria (Dugesia dorotocephala) for mosquito control. J.Amer. Mosq.Control Assoc. 4 (4): 545-546. 1988.

04. FOLHA DE SÃO PAULO (15/07/94). " CE tem suspeita de dengue hemorrágicaSurto atinge 8 em Marilia". Cotidiano 3-3.

05. GERBERG, E.J. \& VISSER, W.M. Preliminary field trial for the biological control of Aedes aegypt by means of Toxorhynchites brevipalpis, a predatory mosquito larva. Mosquito News, 38: 197-200. 1978.

06. HABIB, M.E.M. \& ANDRADE, C.F.S. Bactérias entomopatogênicas In: ALVES, S.B. coord. - Controle Microbiano de Insetos. Ed. Manole, p. 127-170, São Paulo, 1986.

07. KAY, B.H.; CABRAL, C.D.; SLEIGH,A.C; BROWN, M.D.; RIBEIRO, Z.M. \& VASCONCELOS, A.W. Laboratory Evaluation of Brazilian Mesocyclops (Copepoda; Cyclopidae) for mosquito control. J.Med.Entomol. 29 : 599-692, 1992.

08. LACEY, L.A. \& UNDEEN, A.H. Microbial Control of Black flies and Mosquitoes. Ann.Rev. Entomol. 31: 265- 96, 1986.

09. MACEDO, A.C.C.; MELO, A.S. \& ANDRADE, C.F.S. 1994. Avaliação de campo de Dugesia cf. tigrina (Turbellaria, Tricladida) como agente controlador dos vetores da Dengue. IV SICOMBIOL (Simpósio de Controle Biológico), EMBRAPA, Pelotas, R.S., p.275. 1994.

10. MEDINA, F.L; SUAREZ, M.F. \& CLARK, G.G. Aedes aegypt control without chemicals in cemeteries in Puerto Rico. Mosquito Vector Control and Biology in Latin America - A Trird Symposium. J.Amer.Mosq. Control Assoc. 9 (4): 450, 1993. 
SANTOS,L.U.;SOUZA,A.B.;ANDRADE,C.F.S. \& SOUZA,C.E.P. Uso de Bacillus thuringiensis como Agente de controle de Mosquitos em um Cemitério.Rev.Pat.Trop.23 ( 2 ):151-158,jul./dez.1994

11. O'MEARA, G.F.; GETTMAN, A.D.; EVANS Jr.L.F. \& SCHEEL, F.D. Invasion of cemeteries in Florida by Aedes albopictus. J. Amer.Mosq. Control Assoc. 8: 1-10, 1992.

12. O'MEARA, G.F.; EVANS Jr.L.F. \& GETTMAN, A.D. reduced mosquito production in cemetery vases with copper liners. J.Amer. Mosq. Control Assoc. 8 (4): 419-20. 1992.

13. SANTOS, L.U.; CARVALHO, G.A.; DIAS, S.M.G. \& ANDRADE, C.F.S. Potencial de campo de Mesocyclops longisetus (Copepoda, Cyclopidae) como agente controlador de vetores da Dengue. IV SICONBIOL (Simpósio de Controle Biológico), EMBRAPA, Pelotas R.S. Resumos, p. 274. 1994.

14. SCHULTZ, G.W. Cemetery vase breeding of dengue vectors in Manila, Republic of the Philippines. J.Amer. Mosq. Control Assoc. 5: 508-513, 1989. 